\title{
Consideraciones sobre el contenido curricular de matemáticas y su relación con las actitudes
}

\author{
Considerations on the curricular content of mathematics \\ and its relationship with attitudes
}

\author{
Eliel Jiménez Bonilla ${ }^{1}$ \\ William Oswaldo Flores López ${ }^{2}$
}

\section{Resumen}

Esta investigación trata de describir las actitudes hacia las matemáticas, que poseen influencia en la enseñanza y aprendizaje de la disciplina y la necesidad de abordar desde un análisis del contenido curricular de educación secundaria de Nicaragua. Es un estudio cualitativo desde un abordaje de la teoría fundamentada que permitió explicar la teoría y sus relaciones entre las categorías. Por consiguiente, se planteó una guía de preguntas para la revisión de los documentos siguientes: currículo de educación básica y media; programa de matemática de séptimo a undécimo grado; y libro de textos de matemáticas de séptimo a undécimo grado. Se concluye que, existe presencia del componente actitudinal en los contenidos curriculares de la disciplina de matemática, así mismo, componentes emocionales como eje transversal de la educación matemática nicaragüense.

Palabras clave: Contenido Curricular; Actitudes hacia las matemáticas; Emociones, Enseñanza, Aprendizaje.

\section{Abstract}

This research tries to describe the attitudes toward mathematics, that have influence in the teaching and learning of the discipline and the need to approach from an analysis of the curricular content of secondary education of Nicaragua. It is a qualitative study from an approach of the Grounded Theory that allowed to explain the theory and its relations between the categories. Therefore, a question guide was proposed for the revision of the following documents: basic and secondary education curriculum; math program from seventh to eleventh grade; and seventh to eleventh grade math textbook. It is concluded that there is presence of the attitudinal component in the curricular contents of the mathematics discipline, as well as emotional components as a transversal axis of Nicaraguan mathematics education.

1 Licenciado en Ciencias de la Educación con Mención en Matemáticas. Asesor Metodológico del Ministerio de Educación-Rama. Email: ejimenezbonilla@gmail.com

2 Doctor en Educación. Profesor Investigador de la Universidad de las Regiones Autónomas de la Costa Caribe Nicaragüense. Email: william.flores@uraccan.edu.ni (1): https://orcid.org/oooo-ooo2-1016-1620

Recibido: 03/03/2019 Aprobado: 05/05/2019 
Keywords: Curricular content; Attitudes towards mathematics; Emotions, Teaching, Learning.

\section{Introducción}

La problemática relacionada con la enseñanza y aprendizaje de las matemáticas ha sido una preocupación recurrente en varios países. Las actitudes de los estudiantes dentro de este contexto, ha surgido como un elemento relevante que permite explicar algunos aspectos sobre ella. Desde esta perspectiva, surge la necesidad de profundizar en la influencia que el currículo posee en las actitudes de los estudiantes, pues su implementación en el aula podría ayudar a afianzar o modificar las actitudes del estudiantado. En el ámbito educativo se ha tenido un gran interés por analizar y comprender los rasgos cognitivos y de comportamiento que facilitan o ralentizan el desempeño de los estudiantes en cuestiones académicas y cómo se relacionada con las actitudes hacia las matemáticas.

En las investigaciones Flores y Auzmendi (2015), Flores y Olivar-Molina (2016); Jiménez-Bonilla y Flores (2017); y Flores y Auzmendi (2018) se reflejan que las actitudes hacia las matemáticas se relacionan con los factores de la deserción estudiantil, destacando los componentes: emotivos, académicos, económicos, motivacionales, normativos, ambientes familiares, orientación profesional, absentismos por género (femenino), marginación cultural, étnico, lingüísticos, discapacidad y procesos de acompañamiento al estudiantado en su formación. Por consiguiente, el estudio de las actitudes hacia las matemáticas es fundamental porque impiden una progresión en el aprendizaje de las matemáticas.

Por lo anterior expuesto, es de observar que hoy en día el estudio de las actitudes ha tomado mayor fuerza debido a diversos teóricos que se han interesado en evaluar, describir, explicar y correlacionar las variables subyacentes implicadas en este fenómeno. Es por ello, que el objetivo de este artículo es señalar aspectos relacionados con las actitudes hacia las matemáticas que poseen influencia en la enseñanza y aprendizaje de la disciplina y la necesidad de abordarlos desde un análisis del contenido curricular de educación secundaria de Nicaragua.

\section{Literatura}

La dimensión educativa lleva a considerar el conocimiento matemático como una actividad social, propia de los intereses y la afectividad del niño y del joven, cuyo valor principal está en que organiza y da sentido a una serie de prácticas útiles, a cuyo dominio hay que dedicar esfuerzo individual y colectivo. Las investigaciones y estudios internacionales interesados en la mejora curricular y la difusión de las innovaciones, así como la constitución de una comunidad supranacional de educación matemática que trata de superar los aislamientos culturales tradicionales e incorporar las realizaciones 
sociales propias en una corriente de ideas dinámica y progresista, presentan datos que caracterizan el momento actual del currículo de matemáticas dentro del sistema educativo en los países avanzados o en vías de desarrollo (Rico, 1995).

Un currículum es una tentativa para comunicar los principios y rasgos esenciales de un propósito educativo, de forma tal que permanezca abierto a discusión crítica y pueda ser trasladado efectivamente a la práctica (Stenhouse, 1991, p. 29). En su acepción educativa, el concepto de currículo es de origen anglosajón y, en la actualidad, se ha convertido en un término genérico con el que se denomina toda actividad que considere el hecho de planificar una formación. Toda reflexión de carácter curricular contempla, explícita o implícitamente, los siguientes elementos: (1) el colectivo de persona a formar; (2) el tipo de formación que se requiere proporcionar; (3) la institución social en la que se lleva a cabo la formación; (4) las necesidades que se quieren cubrir; y (5) los mecanismos de control y valoración (Rico, 1995).

En cambio, el aprendizaje basado en competencias se fundamenta en un sistema de enseñanza y aprendizaje que progresivamente va desarrollando la autonomía de los estudiantes y su capacidad de aprender a aprender. Así mismo, se basa en un análisis de las exigencias profesionales que ayudarán a definir y priorizar las competencias fundamentales requeridas en una determinada área profesional o de especialidad. Y finalmente, el aprendizaje basado en competencias consiste en desarrollar las competencias genéricas o transversales (instrumentales, interpersonales y sistémicas) necesarias y las competencias específicas (propias de cada profesión) con el propósito de capacitar a la persona sobre los conocimientos científicos y técnicos, su capacidad de aplicarlos en contextos diversos y complejos, integrándolos con sus propias actitudes y valores en un modo propio de actuar personal y profesionalmente (Villa et al., 2007).

En este contexto, se entiende por actitud "una predisposición a actuar de un modo determinado ante un objeto (idea, persona, institución) con una cierta intensidad de modo positivo o negativo" (Villa et al., 2007 p. 19). Para Flores (2019) las actitudes son valoraciones o sentimientos de aceptación o rechazo hacia un constructo u objeto que hombres y mujeres manifiestan en un contexto de resolución de problemas con sus emociones y creencias. Las actitudes se conciben como una predisposición, con cierta carga emocional, que influye en la conducta; es definición remarca tres componentes básicos de la actitud: la cognición o creencias sobre el objeto de esta; el afecto o carga evaluativa de dichas creencias y una interacción de conducta en relación con dicho objeto (Gil, Blanco \& Guerrero, 2005). 


\section{Metodología}

Sabiendo que el enfoque cualitativo utiliza la recolección y análisis de los datos para afinar las preguntas de investigación o revelar nuevas interrogantes en el proceso de interpretación. Además, que la acción indagatoria se mueve de manera dinámica en ambos sentidos: entre los hechos y su interpretación, y resulta un proceso más bien circular en el que la secuencia no siempre es la misma, pues varía cada estudio (Hernández, Fernández \& Baptista, 2014). Se planteo analizar el contenido curricular de matemáticas y su relación con las actitudes en los procesos formación de la educación secundaria de Nicaragua.

Para ello, el abordaje general que se utilizó en este proceso de investigación fue sustentado en la teoría fundamentada porque se trato desarrollar teoría basada en datos empíricos mediante un diseño emergente, es decir, la teoría nace de los datos, sabiendo que, en el diseño emergente se efectúa una codificación abierta y de ésta emergen categorías que se conectan entre si para producir teoría, esto permite a explicar la teoría y sus relaciones entre las categorías. Por ello, se utilizó como método de investigación el análisis de contenido no es otra cosa que una técnica para leer e interpretar el contenido de toda clase de documentos y, más concretamente (aunque no exclusivamente) de los documentos escritos.

Y como técnica, el análisis de documento, porque es una técnica de gran utilidad para obtener información retrospectiva y referencial sobre una situación, un fenómeno o un programa concreto (Del Rincón et al., 1995, 342). Finalmente, se planteo una guía de preguntas para la revisión de los documentos siguientes: currículo de educación básica y media; programa de matemática de séptimo a undécimo grado; y libro de textos de matemáticas de séptimo a undécimo grado.

\section{Resultados}

\section{Las actitudes en el modelo de formación y diseño curricular}

El modelo de formación de formación de las actitudes hacia las matemáticas se efectúa a través del trinomio cuadrado perfecto en que se constituye la educación matemática, es decir, Currículo-Profesor-Estudiante. Elementos básicos que constituyen el modelo de formación de las actitudes, el profesor con su visión de la matemática y de la educación, sus actitudes y creencias; los estudiantes con sus conocimientos, capacidades, creencias, emociones, interacciones en ambientes de aprendizaje; $y$, por último, el currículo configurado con una determinada intencionalidad, es decir, que propone prácticas operativas y discursivas en la resolución de problemas de la vida cotidiana. Véase figura 1. 


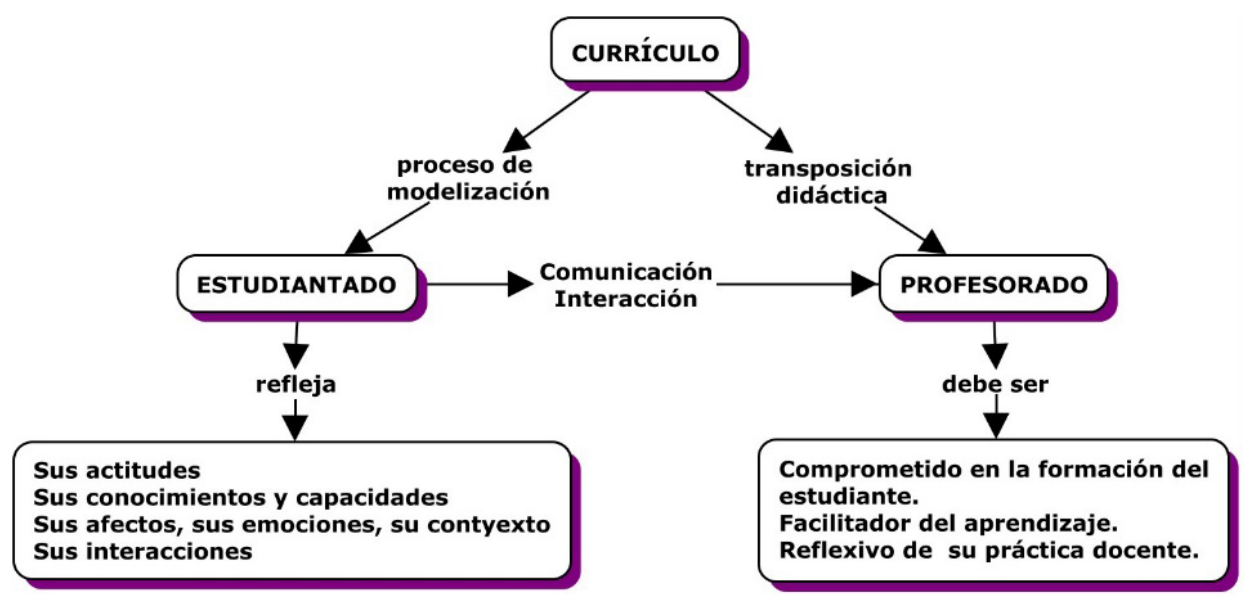

Figura 1: Modelo de formación: currículo-profesor-estudiante.

Entonces, el diseño curricular del subsistema de la educación básica y media nicaragüense (Jarquín-López, 2009), sostiene sobre nuevos pilares, que fortalecen el desarrollo del ser humano en la formación de carácter, autonomía y proyectos de vida.

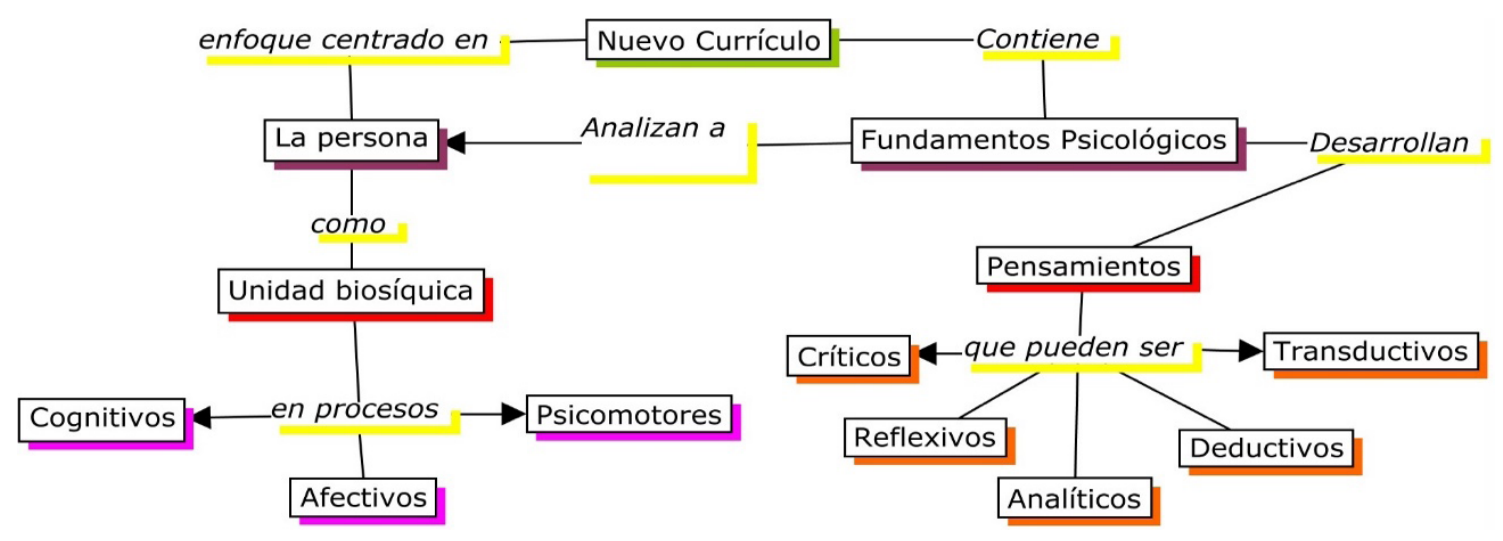

Figura 2: Pilares de la educación básica y media nicaragüense.

Este currículo está organizado en competencias, en áreas y disciplinas para el desarrollo de los aprendizajes, por tanto, orientar la educación hacia el desarrollo de competencias se convierte en una estrategia para formar personas capaces de ejercer sus deberes y derechos, así como para participar en un mundo laboral que requiere, cada vez más, amplios conocimientos. 


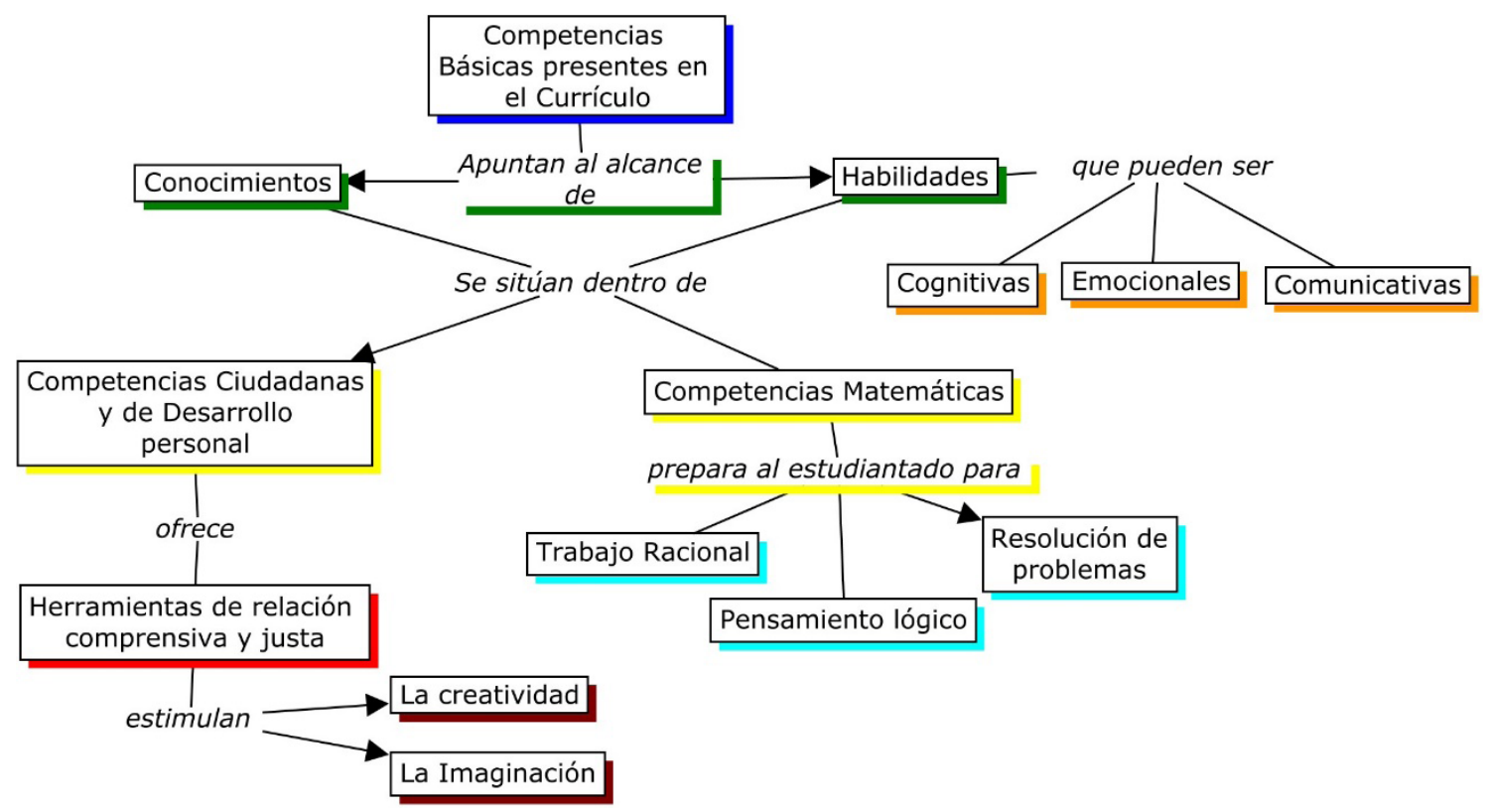

Figura 3: Competencias básicas en el currículo de educación básica y media.

El diseño curricular describe el perfil del docente en el marco de la calidad educativa, tal y como se muestra en la figura siguiente.

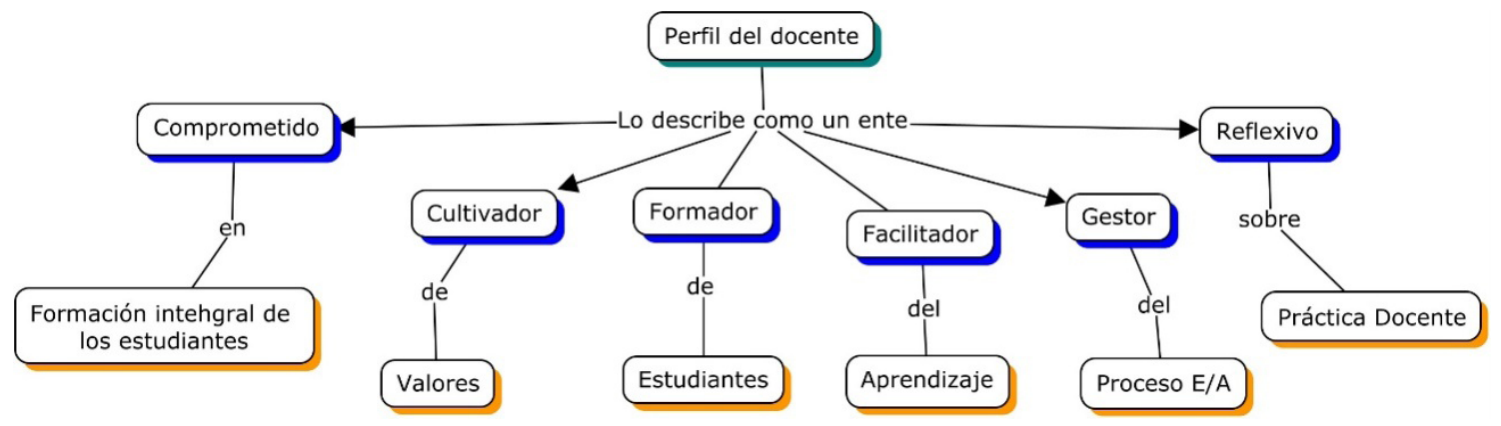

Figura 4: Perfil docente en la educación básica y media.

Este aspecto suele considerarse trascendental en la transmisión y formación de actitudes hacia las matemáticas en el estudiantado, ya que para Martínez (2008), si el docente posee características contrarias a las antes mencionadas, esto da lugar a un frágil y deficiente desempeño profesional que atenta contra la consideración de contenidos actitudinales y otros referentes afectivos que suelen ser relevantes en las decisiones que se tomen en el aula. 


\section{El papel afectivo en los programas de matemática de educación secundaria}

Se ha hecho un análisis exhaustivo en los programas de matemática de educación secundaria en Nicaragua, para evidenciar la presencia del papel afectivo en el desarrollo de los contenidos abordados, durante el proceso de aprendizaje. En dichos programas se orienta el desarrollo del dominio afectivo en el abordaje de distintos contenidos matemáticos pertenecientes a los pensamientos matemáticos (estadístico - probabilístico, numérico - algebraico, y geométrico), con la finalidad que el estudiantado pueda apropiarse de actitudes positivas que posibilite un cambio en las creencias y expectativas hacia la materia y favorecer su acercamiento hacia ésta.

Tabla 1: El papel afectivo en los pensamientos matemáticos

\begin{tabular}{|c|c|}
\hline PENSAMIENTOS & ACCIONES \\
\hline $\begin{array}{l}\text { Pensamiento Estadístico } \\
\text { probabilístico }\end{array}$ & $\begin{array}{l}\text { - Estimular el interés, talentos, habilidades y destrezas cognitivas y motrices. } \\
\text { - Comprender las propias emociones y sentimientos. } \\
\text { - Valorar en el estudiantado el pensamiento Creativo. } \\
\text { - Establecer y mantener relaciones interpersonales, significativas y respetuosas de su } \\
\text { entorno. } \\
\text { - Practicar la automotivación y autorregulación. } \\
\text { - Participar en actividades donde se desarrollen los talentos, las habilidades, pensa- } \\
\text { mientos creativos, el alcance de los logros personales, fortalecimiento de la autoes- } \\
\text { tima y la identidad personal. }\end{array}$ \\
\hline $\begin{array}{l}\text { Pensamiento Numérico } \\
\text { Algebraico }\end{array}$ & $\begin{array}{l}\text { - Mantener la motivación e instar a la participación durante la clase, para tomar } \\
\text { conciencia de la vocación hacia el estudio. } \\
\text { - Manifestar pensamientos, emociones, sentimientos, deseos y necesidades de } \\
\text { forma asertiva. } \\
\text { - Convivir en armonía, para lograr la satisfacción de las necesidades psicosociales. } \\
\text { - Desarrollar comunicación crítica y reflexiva que fomenten el sentido de valía y la } \\
\text { capacidad de ser o hacer. }\end{array}$ \\
\hline Pensamiento Geométrico & $\begin{array}{l}\text { - Fomenta la toma de decisiones acertadas que le permita alcanzar el logro de sus } \\
\text { metas y objetivos a nivel personal, escolar y familiar. } \\
\text { - Apreciar y valorar el dominio de las y los estudiantes sobre la aplicación de teore- } \\
\text { mas en la solución de problemas. } \\
\text { - Muestra confianza en su propia capacidad para comprender las relaciones espacia- } \\
\text { les y resolver situaciones reales. }\end{array}$ \\
\hline
\end{tabular}

\section{El planeamiento didáctico de las actitudes hacia las matemáticas}

El planeamiento didáctico de las actitudes hacia las matemáticas implica fundamentalmente procesos de previsión, selección y organización de todos los elementos que componen la situación del proceso enseñanza-aprendizaje. Es importante que el o la docente, antes de que concrete su planeamiento, se plantee algunas interrogantes que 
le aclaren sobre la manera más efectiva en que puede desarrollar su práctica pedagógica. En este contexto, el profesorado de matemática realiza el planeamiento didáctico utilizando indicadores de logro, contenidos básicos y estrategias metodológicas: Que se tipifican en aspectos como:

- Indicador de logro: aquí es importante incluir cuáles son los aprendizajes esperados que se propone desarrolle el estudiantado, una vez que concluya la clase.

- Contenidos básicos: estos se derivan de los indicadores de logro, ya que son un medio para alcanzar los aprendizajes esperados. Estos son el medio para desarrollar los indicadores de logro y competencias de grado.

- Estrategias metodológicas: acá se incluyen las situaciones de aprendizajes que desarrollará en el aula de clase, es decir todas las actividades vinculadas con los contenidos y con los ejes transversales del currículo, las estrategias metodológicas que promueven la motivación del estudiantado, de manera que ésta sea dinámica, atractiva y participativa.

En el planeamiento didáctico el profesorado se plantea interrogantes sobre la mejor manera en que pueden desarrollar su práctica pedagógica de forma efectiva, para ello parte de la reflexión que se visualiza en la tabla siguiente:

Tabla 2: Interrogante en el proceso de planificación de las actitudes

\begin{tabular}{|c|c|}
\hline Pregunta a reflexionar & Estrategia Pedagógica \\
\hline ¿Qué está pasando? & Diagnóstico \\
\hline ¿Qué se quiere hacer? & $\begin{array}{l}\text { Elaboración de actividades-acciones a realizar considerando el apoyo al mo- } \\
\text { mento del proceso y su secuencia. }\end{array}$ \\
\hline ¿Cómo se va a hacer? & $\begin{array}{l}\text { Actividades metodológicas para alcanzar las competencias y los indicadores } \\
\text { de logro. }\end{array}$ \\
\hline $\begin{array}{l}\text { ¿Con quiénes se va a hacer y a quiénes va } \\
\text { dirigido? }\end{array}$ & Con grupos de docentes y estudiantes. \\
\hline ¿Con qué se va a hacer? & $\begin{array}{l}\text { Recursos didácticos, deben consultarse los Programas de estudio, guías didác- } \\
\text { ticas, antologías, libros de texto, recursos tecnológicos }\end{array}$ \\
\hline ¿Cuánto tiempo se requiere para hacerlo? & $\begin{array}{l}\text { Tiempo necesario para desarrollar su Plan Didáctico de acuerdo con la progra- } \\
\text { mación del TEPCE }\end{array}$ \\
\hline ¿Dónde lo realizará? & (Aula, patio, biblioteca, comunidad, otros) \\
\hline ¿Cómo se evaluará? & Conforme a lo programado \\
\hline
\end{tabular}

\section{Discusión y conclusiones}

En este artículo se presentan algunas consideraciones sobre el contenido curricular de matemáticas y su relación con las actitudes. El currículo de educación básica y 
media presenta el componente actitudinal como contenidos curriculares que incluyen actitudes; valores y normas, con el propósito de fortalecer la función moral o ética de la educación. Además, puede incluirse tres tipos de actitudes:

- Actitudes hacia los contenidos conceptuales (interés o curiosidad por conocer el medio ambiente, actitud indagadora ante la realidad, actitud crítica ante los hechos sociales);

- Actitudes y valores comunes a un conjunto de áreas o disciplinas, los que se ven como guías para el aprendizaje (cuidado en el uso de materiales, orden y aseo en el trabajo, gusto por el trabajo compartido);

- y un conjunto de actitudes específicamente morales, ambientales que tienen carácter más transversal que específico de un área (sensibilidad y respeto por el medio ambiente, respeto a la opinión ajena).

En este contexto, el estudiante debe desarrollar habilidades, destrezas, aptitudes, actitudes y valores, que le propicie un pensamiento crítico, creativo, imaginativo, espacial y lógico, para adaptarse en el medio, actuar con autonomía y seguir aprendiendo para mejorar su calidad de vida.

Por otra parte, el currículo de educación básica y media promueve la inteligencia emocional, definiéndola, el talento o capacidad de la persona para percibir, aplicar, comprender, controlar o manejar sus propias emociones y sentimientos y entender el de los demás, para ser más efectivos en las relaciones interpersonales y que les permita vivir y convivir en un ambiente de paz. La inteligencia emocional integra aptitudes personales (el autoconocimiento, la autorregulación y la motivación) y aptitudes sociales (habilidades sociales). Es por ello, que Jarquín-López (2009) plantea que es necesario:

- Comprender las propias emociones y sentimientos, así como su efecto en las demás personas.

- Manifestar pensamientos, emociones, sentimientos, deseos y necesidades de forma asertiva, a fin de sentirse bien consigo mismo o misma y resolver de forma pacifica las diferencias.

- Controlar emociones y sentimientos que les permita saber canalizarlas correctamente sin afectar a las demás personas.

- Interpretar y respetar las emociones de las demás personas, a fin de establecer relaciones sociales y vínculos personales más armónicos.

- Practicar la automotivación y la autorregulación para controlar la impulsividad y responder de forma adecuada a las exigencias del medio donde se desenvuelve.

- Mostrar conductas positivas y cualidades, que facilite el dominio de la comunicación y el autocontrol emocional para fortalecer la confianza en sí mismo/a. 
Todas ellas necesarias para conducirse y hacer frente a las situaciones adversas de la vida. Es relevante identificar el papel afectivo en el currículo de educación secundaria porque constituyen una información valiosa tanto para el diseño, planificación e implementación de los procesos de enseñanza por parte del profesorado, como para el desarrollo de los procesos formativos a generar en el estudiantado, quienes van a seguir interactuando con dicha disciplina no solo en el ámbito académico, sino también en el profesional.

\section{Lista de referencias}

Del Rincón, D., Latorre, A., \& Arnal, J. (1995). Técnicas de investigación en Ciencias Sociales. Madrid: Ed. Dykinson.

Flores W., O. (2018). Actitudes hacia las matemáticas y su relación con investigación propia de resolución de problemas.

Flores, W. O. \& Auzmendi, E. (2018). Actitudes hacia las matemáticas en la enseñanza universitaria y su relación con las variables género y etnia. Profesorado. Revista de Currículum y Formación de Profesorado, 22(3), 231-251. DOI: https:// doi.org/10.30827/profesorado.v22i3.8000

Flores, W. O., \& Olivar-Molina, S. (2016). Actitudes hacia la estadística en la formación del profesorado para contextos multiculturales. Revista Universitaria Del Caribe, 17(2), 27-37. DOI: https://doi.org/10.5377/ruc.v17i2.3235

Flores, W., O., \& Auzmendi, E. (2015). Análisis de la estructura factorial de una escala de actitud hacia las matemáticas. Aula de Encuentro, 17(1), 45-77.

Gil, N., Blanco L., \& Guerrero, E. (2005). El dominio afectivo en el aprendizaje de las matemáticas. Una revisión de sus descriptores básicos. UNIÓN. Revista Iberoamericana de Educación Matemática, 2, 15-32.

Hernández, R., Fernández, C., \& Baptista, M. (2015). Metodología de la Investigación. México: McGraw-Hill.

Jarquín-López, H. (2009). Programa de Estudio de Matemáticas de Educación Secundaria (7mo, 8vo y 9 mo grado).

Jiménez-Bonilla, E., \& Flores, W., O. (2017). Actitudes hacia las matemáticas: un estudio en una escuela rural de la Costa Caribe Sur de Nicaragua. Revista Universitaria Del Caribe, 18(1), 7-16. DOI: https://doi.org/10.5377/ruc.v18i1.4794 
Martínez, O. (2008). Actitud hacia la matemática. Sapiens: Revista Universitaria de Investigación, 237-256.

Rico, L. (1995). Consideraciones sobre el currículo escolar de Matemáticas. Revista EMA, 1(1), 4-24.

Stenhouse, L. (1991). Investigación y desarrollo del curriculum. Madrid: Ed. Morata.

Villa, A., \& Villa, O. (2007). El aprendizaje basado en competencias y el desarrollo de la dimensión social en las universidades. Educar, 40, 15-48. 